\title{
8. Konventionalisierung und Trivialisierung der Gattung: Zur Rolle der Epigonen
}

Um die einzelnen Aspekte der kulturspezifischen Indienstnahme des Textmodells Prosagedicht im deutschsprachigen Raum anschaulich darstellen zu können, hat sich der hier vorgelegte Überblick über die Gattungsgeschichte bislang auf mehr oder weniger prominente Einzelpersonen konzentriert. Eine solche Fokussierung auf namhafte Autoren tendiert freilich dazu, das Gesamtbild an einigen Stellen zu verzerren. So könnte etwa der Umstand, daß einige der bedeutenden Schriftsteller der Ausdrucksform mit Vorbehalten begegneten bzw. sie nur kurzzeitig nutzten, leicht den Eindruck erwecken, das Prosagedicht sei - alles in allem genommen - letztlich nur eine Randerscheinung der Literaturgeschichte. Tatsächlich jedoch stellt diese Gattung, wie ein Blick in die einschlägigen Literaturzeitschriften zeigt, um die Jahrhundertwende ein regelrechtes Massenphänomen dar. Nahezu in jedem renommierten Journal, sei es nun die Gesellschaff, die Freie Bühbne für modermes Leben/Freie Bübne für den Entwickelungskampf der Zeit/Neue deutsche Rundschau/Neue Rundschau, die Modernen Blätter, die Gegenwart, Das litterarische Echo, Pan, Jugend, die Zukunft, der Sturm, die Aktion, Brenner, Daimon, das Neue Pathos oder die Weißen Blätter, finden sich Kurzprosatexte, die sich jenseits traditioneller Vertextungskonventionen bewegen; nicht wenige davon sind durch Titel oder Untertitel bzw. durch den Veröffentlichungskontext (z.B. die Zuweisung zu Rubriken wie "Gedichte" oder wLyrisches") eindeutig als Prosagedichte gekennzeichnet. Dazu kommen noch all jene Texte, die gemischt mit Versgedichten bzw. Kurzprosa in Einzelpublikationen eines Autors oder auch in Sammelbänden mit Beiträgen mehrerer Autoren abgedruckt wurden.

Allerdings sind die Namen vieler Schriftsteller, die sich um 1900 der Textsorte Prosagedicht bedienten, heute nur noch wenig oder gar nicht mehr bekannt; von manchen lassen sich nicht einmal grundlegende biographische Daten ermitteln. Für den Gesamtzusammenhang der Gattungsgeschichte sind diese poetae minores aber insofern bedeutsam, als sic Verständnis und Einschätzung des Genres in der Wahrnehmung der Zeitgenossen zumindest mitgeprägt haben. Ihnen kommt also eine entscheidende Rolle bei jenem Prozeß zu, den Nies einmal treffend als "standardisation de l'exceptionnel en littérature « bezeichnet hat. Einer breiteren Öffentlichkeit

1 Fritz Nies: Des Amadis aux Zöles. Standardisation de l'exceptionnel en littérature. In: Normes et transgressions. Actes du colloque franco-allemand de Nantes, 14-16 mars 1984. 
ist das Phänomen Prosagedicht nämlich sicher erst durch die zahlreichen Nachahmer ins Bewußtsein gerückt worden, die das bis dato kaum bekannte Vertextungsmodell aufgriffen und auf dem Weg der Imitation verbreiteten. Damit veränderte sich aber auch dessen Wertstatus, weil unter den Bedingungen des modernen Literatursystems jede zusätzliche Indienstnahme einer Ausdrucksform deren Entwertung bedeutet. ${ }^{2}$ Was die Epigonen also für eine kommunikationsgeschichtlich orientierte Darstellung der Gattungsentwicklung interessant macht, ist die durch sie machtvoll in Gang gesetzte Dynamik von Exklusivitätsverlust und Popularitätsgewinn, welche sowohl den Konstitutions- als auch den sich daran anschließenden Evolutionsprozeß des Genres entscheidend beeinflußt.

Bourdieu hat gezeigt, daß das moderne Funktionssystem der Kunst sich in ein Feld mit unterschiedlichen Polen ausdifferenziert: den Sektor der »Massenproduktion « und den der weingeschränkten Produktion» ${ }^{3}$. Während ersterer den Beteiligten finanziellen Gewinn verspricht, die Kulturschaffenden aber zugleich in den Bereich kommerzieller Gebrauchskunst verweist, verlangt ersterer den Künstlern einen weitgehenden Verzicht auf materielle Gratifikationen ab, entschädigt sie im Gegenzug freilich durch die Akkumulation von ssymbolischem Kapitak. Im Segment der spezialisierten Produktion von Kulturgütern mit whohem Weihegrad “ spielt die Marktgängigkeit eines Artikels keine nennenswerte Rolle, ja sie erweist sich letztlich sogar als schädlich für dessen Ansehen. Viel wichtiger sind dafür symbolisch codierte binnenliterarische Distinktionskriterien, die nur von einem ausgewählten Rezipientenkreis als solche wahrgenommen und bewertet werden können. Weil die Autoren ständig ihre Hervorbringungen im Interferenzbereich von merkantilem Ertrag und künstlerischer Wertschätzung und damit auf einer andauernden Schwankungen ausgesetzten doppelten Skala ökonomischer und symbolischer Wertschätzung verorten müssen, werden sie unter den Bedingungen der Moderne notwendig zu Medienund Textstrategen. Der Gattungswahl kommt dabei eine herausragende Bedeutung zu, weil sie in vielen Fällen entscheidende Hinweise gibt, wo der jeweilige Text im literarischen Feld zu verorten ist:

À la mémoire de Pierre Dumonceaux. Éd. par Daniel Briolet. Nantes: Université de Nantes 1986 (= Textes et Langages 12), S. 95-104.

2 Bourdieu hat dies auf die griffige Formel gebracht: Nachfrage«; Pierre Bourdieu: Die Regeln der Kunst. Genese und Struktur des literarischen Feldes, S. 345. Da in der Modeme sdas Ausmaß an Unabhängigkeit von der Nachfrage des breiten Publikumsı und den vom Markt ausgehenden Zwängenı in direkter Weise mit der Reputation der Kulturproduzenten korreliert, ließen vor allem die auf Distinktion bedachten Autoren das Textmuster Prosagedicht rasch wieder fallen, was seinem Ruf natürlich schadete; ebd.

3 Ebd., S. 344.

4 Vgl. hierzu die beiden graphischen Überblicksdarstellungen zum Feld der kulturellen Produktion allgemein und zur Beschaffenheit des literarischen Feldes im späten 19. Jahrhundert bei Pierre Bourdieu: Das literarische Feld, S. 48 und 104. 
Die Gattungen [...], die meiste Zeit stillschweigende und stillschweigend von allen der im [literarischen] Feld anerkannten Akteure anerkannte Gesamtheiten von Gepflogenheiten und Vorannahmen, die den Entwurf und die Wahmehmung entsprechender Werke strukturieren, legen auch die Stellungen fest, die den verschiedenen Akteuren als annehmbar oder unannehmbar, verlockend oder abstoßend erscheinen können ${ }^{5}$.

Das Prosagedicht nimmt in diesem Zusammenhang eine ambivalente Stellung ein: Als neue und damit unverbrauchte Vertextungsform, die zudem aus dem Ausland kommt und den Reiz des Fremden mit sich trägt, verfügt sie einerseits über jenes symbolische Kapital, das sie zu einem begehrten Gestaltungsmittel elitärer Künstler werden läßt. Andererseits verweisen sie ihre Gattungsmerkmale eindeutig in den Bereich der ästhetisch als tendenziell minderwertig angesehenen Prosa, ${ }^{6}$ was sie für an der literarischen Werthierarchie orientierte Autoren eher uninteressant macht. So verbinden sich im Genre Prosagedicht also Elemente, die Distinktionsgewinn versprechen mit solchen, die eher einen Prestigeverlust befürchten lassen. Dieser zwiespältige symbolische Stellenwert dürfte im übrigen der Grund dafür sein, warum in Deutschland - anders als etwa in Frankreich - bereits etablierte Autoren die Gattung mieden, weil sie durch eine Nutzung nur unnötigerweise ihre bereits erreichte Reputation gefährdet hätten.

Anders sah die Lage indes für Schriftsteller aus, die sich erst einen Platz im Literaturbetrieb erkämpfen mußten. Sie hatten (noch) kein symbolisches Kapital zu verspielen und konnten (bzw. mußten) sich daher risikofreudiger verhalten. Da die wichtigsten Positionen des Marktes bereits besetzt waren, bekam für sie die Suche nach Nischen eine herausgehobene Bedeutung. Ihnen nun bot das Prosagedicht eine willkommene Chance, sich als ästhetische Neuerer zu profilieren und in dieser Rolle öffentlich wahrgenommen zu werden. Und so wurde die Auseinandersetzung mit dem Prosagedicht für nahezu sämtliche sich als Lyriker verstehenden Literaten, die in den neunziger Jahren debütierten, zum Ausweis ihrer Modernität und zum beliebten Vehikel für die Schaffung eines eigenen Autorprofils. Intensiv genutzt wurde die neue Gattung zwar vor allem von Schriftstellern, die ihre Wurzeln noch in der - dem Ausdrucksmodus Prosa gegenüber aufgeschlossenen - Poetik des Naturalismus hatten, aber selbst betont antinaturalistisch ausgerichtete Autoren mit einer

\footnotetext{
Ebd., S. $120 \mathrm{f}$.
}

6 Noch in der Konstitutionsphase des Naturalismus in Deutschland sieht sich ein im Literaturbetrieb etablierter und weithin respektierter Lyriker wie Robert Hamerling genötigt, die Veröffentlichung eines Bandes mit »Prosaaufsätzen» zu rechtfertigen. Er rekapituliert dabei die gängigen Klischees, denen sich die zeitgenössischen Autoren ausgesetzt sahen: „Es gibt Verehrer und Verehrerinnen der Poesie, welche glauben, daß der Poet, der sich für gewöhnlich in gebundener Rede vernehmen läßt, heruntersteigt und sich etwas vergibt, wenn er Prosa schreibt. [...] Es gibt auch Solche, die sich vorstellen, wenn ein Dichter (im engeren Wortsinn) Prosa schreibe, so thue er es so nebenbei und gelegentlich, auf äußeren Antrieb, werfe so leichte Waare gleichsam zur Erholung hin.« Robert Hamerling. Prosa. Skizzen, Gedenkblätter und Studien. Bd. 1. Hamburg. J.F. Richter 1884, S. IX. 
deutlichen Präferenz für die Versdichtung konnten und mochten sich den Möglichkeiten des Genres nicht entziehen, zumal seine Herkunft aus dem französischen Symbolismus es für sie gleichfalls, wenn auch aus anderen Gründen interessant erscheinen ließ.

Die forcierte Indienstnahme des Prosagedichts durch literarische Debütanten und seine Instrumentalisierung zu Zwecken auktorialer Selbstprofilierung hatte allerdings erhebliche Rückwirkungen auf den Status der Gattung in Deutschland und Österreich. Während die Prosagedichte Baudelaires und Turgenevs (mit gewissen Einschränkungen auch die Mallarmés) dezidiert als Spätwerke zu bezeichnen sind und jene Rimbauds immerhin zum Letzten gehören, was der - zugegeben noch sehr junge - Autor vor seinem literarischen Verstummen geschrieben hat, stammen die entsprechenden Texte deutschsprachiger Schriftsteller durchweg aus der Anfangsund Erprobungsphase ihrer Autorschaft. Dieser Umstand führt dazu, daß sich der symbolische Wertstatus der Gattung im jeweiligen kulturellen Kontext radikal unterscheidet: Einmal erscheint das Genre auf Grund seiner Stellung in der Werkchronologie als Konsequenz und Summe eines CEuvres - bei Baudelaire und Turgenev sogar als textuelles Vermächtnis -, wodurch es eine enorme Aufwertung erfährt, das andere Mal verleiht die frühe Entstehung der Texte und die Tatsache, daß viele Autoren anschließend andere, meist konventionelle Ausdrucksformen bevorzugten, ihm den Charakter eines Durchgangsstadiums, das - um der weiteren literarischen Entwicklung willen - überwunden werden muß. Vollends die zahlreichen poetae minores, die vom Dinstinktionsgewinn, den die rasch modisch gewordene Gattung versprach, profitieren wollten, diskreditierten das Prosagedicht nachhaltig. ${ }^{7}$ Indem sie sich an Leitfiguren der Genreentwicklung orientierten und deren Verfahrensweisen im Umgang mit dem Textmodell mehr oder weniger umstandslos nachahmten, trugen sie zur vorzeitigen Automatisierung seiner Ausdrucksmöglichkeiten entscheidend bei.

Welche Folgen die Prägekraft eines einzigen Autors auf Akzeptanz und Handhabung einer Gattung haben kann, zeigt sich schon an Turgenev sehr deutlich, ${ }^{8}$ mehr aber noch an Detlev von Liliencron, der ja das Prosagedicht in die deutsche Literaturgeschichte eingeführt hat. Sein Beispiel rief eine ganze Reihe von mehr oder weniger talentierten Nachahmern auf den Plan, welche die von ihm praktizierten Neue-

7 Ortlieb irrt also, wenn sie meint, daß die mkurze Prominenz der neuen Gattung die Argumente ihrer theoretischen Negierung nicht beeinflusstu habe; Comelia Ortlieb: Poetische Prosa. Beiträge zur modemen Poetik von Charles Baudelaire bis Georg Trakl, S. 279.

8 Siehe in diesem Zusammenhang besonders Otto Kemmer: Gedichte in Prosa. Aus den Papieren eines Pessimisten. Berlin: In Commission der Kamlah'schen Buchhandlung (Georg Nauck) 1885, Anatole Rembe: Hieroglyphen. Leipzig: Wilhelm Friedrich 1886, und Fritz Mauthner. Lügenohr. Fabeln und Gedichte in Prosa. Stuttgart: Verlag der J.G. Cotta'schen Buchhandlung Nachfolger 1892 (zweite Auflage unter dem Titel: Aus dem Märchenbuch der Wahrheit. Stuttgart: Verlag der J.G. Cotta'schen Buchhandlung Nachfolger 1896). 
rungen rasch auf minderem Niveau popularisierten. $\mathrm{Zu}$ diesen Epigonen - und deren Schülern -, welche die bevorzugten literarischen Themen und Formen des Vorbilds aufgriffen und varïerten, zählen neben Bierbaum und Croissant-Rust, die sich immerhin eine gewisse Eigenständigkeit zu erschreiben vermochten, vor allem $\mathrm{Gu}$ stav Falke (1853-1916) ${ }^{9}$, Carl Busse $(1872-1918)^{10}$ und Wilhelm Holzamer (1870$1907)^{11}$, in gewisser Weise auch Wilhelm Arent $\left({ }^{*} 1864\right)^{12}$, Alfred Guth $\left({ }^{*} 1875\right)^{13}$, Hans Bethge (1876-1946) ${ }^{14}$ und Heinrich von Schullern $(1865-1955)^{15}$. Sie alle bedienten sich der von Liliencron etablierten Verwendungsweisen des Prosagedichts

9 Vgl. Gustav Falke: Mynheer der Tod und andere Gedichte. Dresden/Leipzig: Pierson 1892; ${ }^{2} 1896$. Daß »das Buch sichtlich unter dem Einfluß Liliencrons stehtw, verraten der unverhohlen auf den Band Adjutantenritte und ander Gedichte (1883) anspielende Titel ebenso wie die Widmung an Liliencron; Ernst Ludwig Schellenberg. Gustav Falke. Eine Studie. Leipzig: Verlag für Literatur, Kunst und Musik 1908 (= Beiträge zur Literaturgeschichte 55), S. 10. Doch damit nicht genug: "Auch die nach Liliencrons Muster die Verse gelegentlich ablösenden lyrischen Prosaskizzen zeigen [...] das Vorbild«; Heinrich Spiero: Gustav Falke. Ein Lebensbild. Mit zwei Bildtafeln und einer unveröffentlichten Handschrift. Braunschweig/Berlin/Hamburg: Georg Westermann 1928, S. 25. Zwei der insgesamt drei in Mynheer der Tod enthaltenen Prosagedichte nahm der Autor später in seine Gesammelten Dichtungen auf; vgl. Gustav Falke: Gesammelte Dichtungen. Bd. 4: Der Schnitter. Gedichte. Hamburg/Berlin: Alfred Janssen 1912, S. 10-12. Im Rückblick hat Falke selbst darauf hingewiesen, daß Liliencrons Texte wie seine Offenbarung für ihn gewesen seien; Fritz Bökkel: Detlev von Liliencron. Erinnerungen und Urteile, S. 51. Beide Autoren standen zudem in persönlichem Kontakt: Nachdem sie einander im Frühjahr 1891 persönlich kennengelernt hatten, fungierte Liliencron schon wenige Monate später als Taufpate für Falkes erste Tochter Gertrud. Die wachsende Verbundenheit mit dem »Freund" (L-GW VIII, S. 335) drückt sich schließlich am sinnfälligsten darin aus, daß Liliencron seinen Nexen Gedichten (1893) eine lyrische Widmung an Falke voranstellt. Vgl. Carl Busse: In junger Sonne. Novellen und Skizzen. München: Poeßl 1892 (S. 223250: "Gedichte in Prosak) sowie C. B.: Gedichte in Prosa. In: Jugend. Münchner illustrierte Wochenschrift für Kunst und Leben 1 (1896), S. 298 (enthält die Texte Die Insel der Seligen und Enzartung). Der Autor hat über sein Vorbild einen Aufsatz verfaßt; vgl. Carl Busse: Detlev von Liliencron. In: Blätter für literarische Unterhaltung, 1896, Teil 2, S. 498f. Soergel bezeichnet Busse als "Eklektiker» und unterstreicht: "von Modemem hat Liliencrons frische Bilderwelt am meisten die Phantasie Carl Busses befruchtetc; Albert Soergel: Dichtung und Dichter der Zeit. Eine Schilderung der deutschen Literatur der letzten Jahrzehnte, S. 511 und 512 .

11 Vgl. Wilhelm Holzamer: Auf staubigen Straßen. Skizzen. Berlin/Leipzig. Schuster \& Loeffler 1898. Holzamer, der mit Falke win herzlicher Freundschaft verbunden« war, stand auch literarisch unter dessen Einfluß; Gustav Falke: Die Stadt mit den goldenen Türmen. Die Geschichte meines Lebens. Berlin: G. Grote'sche Verlagsbuchhandlung 1912, S. 467. So widmete er ihm seinen Band Zum Licht (1897). Vgl. in diesem Zusammenhang auch Falkes Nachruf auf Holzamer im Litterarischen Echo.) "Liliencrons Einfluß kommt zu Holzamer auf dem Umweg über Falke.» Günter Heinemann: Wilhelm Holzamer. Persönlichkeit und Schaffen (1870-1907). Idstein i.'Ts.: Grandpierre 1956, S. 35. Des weiteren läßt sich auch eine Affinität zu Flaischlen feststellen; vgl. etwa Wilhelm Holzamer: Cäsar Flaischlen. In: Das litterarische Echo 4 (1901/02), Sp. 1596-1601. Siehe in diesem Zusammenhang auch 
und präsentierten dem Publikum entweder Sammclbände mit eher narrativ geprägter Kurzprosa oder Mischpublikationen von Vers- und Prosatextcn.

Abgelöst wurde Liliencron als bevorzugtes Bezugsmodell erst in der zweiten Hälfte der neunziger Jahre. Die allgemein um sich greifende Tendenz zur Lyrisierung, weiche auch den Spielarten spoetischer Prosar zu neucr Attraktivität verhalf, ließ nun rhetorisierte und rhythmisierte Prosagedichte, wie Bierbaum und CroissantRust, mehr aber noch Dauthendey und Schlaf sie verfaßten, nachahmenswert erscheinen. Dieser Paradigmenwandel schlug sich nicht zuletzt in der Benennung der veröffentlichten Texte nieder, und so rückte an die Stelle des immer noch der Ästhetik der Prosa verhafteten Terminus ,Skizzer als neuer, auf den Bereich der ,Poesiec zielender Leitbegriff das Programmwort Dichtungt. Belege hierfür sind zwei Bände von Paul Bornstein (1868-1939) ${ }^{16}$ und Franz Himmelbauer (1871-1918) ${ }^{17}$. In allen genannten Beispielen blieb es freilich jeweils bei einer derartigen Buchveröffentlichung. Nachdem die Autoren mit der - gemessen am Erscheinungsbild der meisten

Karl Neurath: Wilhelm Holzamer. Leipzig: Verlag für Literatur, Kunst und Musik 1910 (= Beiträge zur Literaturgeschichte 59) und Adalbert Schmidt: Wilhelm Holzamer. Sein Leben und sein Werk. Diss. (Masch.) Wien 1930.

12 Vgl. Wilhelm Arent: Aus dem Großstadtbrodem. Mit cinem Geleitwort von Lucian von Prager. Zürich: Verlags-Magazin 1891. In seiner Vorbemerkung kommt von Prager exlizit auf den Experimentcharakter der in Arents Band enthaltenen Prosagedichte zu sprechen, verweist dabei aber auf die Anregerwirkung Turgenevs: "Seltsam gemischt ist der erste Eindruck, den man bei der Lektüre von diesen (in der Art von Turgenjew's Gedichten in Prosa) gehaltenen Skizzen empfängt, und es bleibt abzuwarten, wie sich die Kritik zu diesen, allerdings das prae absoluter Originalität für sich habenden Versuchen stellen wird. « Ebd., S. XI.

13 Vgl. Alfred Guth: Vom Wege. Skizzen. Leipzig: Wilhelm Friedrich o.]. [1897].

14 Vgl. Hans Bethge: Syrinx. Ein Skizzenbuch. Breslau: Schottlacnder 1898. Im Hinblick auf Liliencron gestand Bethge »die Verehrung, die ich seit langem für ihn habew, freimütig ein; Fritz Böckel: Detlev von Liliencron. Erinnerungen und Urteile, S. 20. Der Autor gab u.a. eine Gedichtanthologie mit dem Titel Deutsche I yrite seit Liliencron (Leipzig: Hesse 1905) heraus. Interessanterweise bezeichnet Bethge in seiner Rezension von Else Lasker-Schülers Prosaband Die Nächte Tino ton Bagdads (1907) die cinzelnen Abschnitte des Buchs als "Gedichte in Prosa«; Das litterarische Echo 10 (1907/08), Sp. 959.

15 Vgl. Heinrich von Schullern: Helldunkel. Bilder und Lieder. Wien: Lesk \& Schwidernoch 1892 sowie H. v. S.: Neues Skizzenbuch. Linz/Wien/Leipzig: Österreichische Verlagsanstal 1900. Zu Leben und Werk dieses Autors siehe vor allem Karl Paulin: Heinrich von Schullern und seine Zeit. Ein Lebensbild des österreichischen Dichters. Innsbruck: Wagner 1960.

16 Vgl. Paul Bornstein: Aus Dämmerung und Nacht. Gedichte und Prosadichtungen. Braunschweig: C.A. Schwetschke und Sohn 1896. Bomstein übernimmt zwar das Prinzip der Mischpublikation, separiert aber innerhalb seines Bandes die Texte in die Rubriken »Gedichter und "Gedichte in Prosau. Daß er Croissant-Rusts Gedichte in Prosa (1893) kannte, geht aus einem Artikel über die Autorin hervor; vgl. Paul Bornstein: Anna Croissant-Rust. In: Das litterarische Echo 9 (1906/07), Sp. 924-933.

17 Vgl. Franz Himmelbauer: Waldsegen. Prosadichtungen. Linz/Wien/Leipzig: Österrcichische Verlagsanstalt 1900; München/Lcipzig: Georg Müller 0.J. Greinz zählt Himmclbauer 
anderen Neuerscheinungen nach wie vor ungewöhnlichen - Machart ihrer Textsammlungen auf positive oder negative Art Aufsehen erregt und sich einen gewissen Bekanntheitsgrad verschafft hatten, zogen sie es durchweg vor, auf anerkannte literarische Gestaltungsmuster zurückzugreifen. Profilierungsstrategisch verhielten sie sich damit durchaus klug, weil sie so ihre schriftstellerische Seriosität glaubwürdig unter Beweis stellten. Der Gattung Prosagedicht verschaffte eine derart kurzfristiginstrumentelle Nutzung dagegen den Nimbus einer vor allem bei Anfängern beliebten Vertextungspraxis, mit deren Hilfe unter Umständen zwar kurzfristig Aufsehen zu erregen war, deren momentane Skandalisierungswirkung sie allerdings für etablierte Schriftsteller weitgehend inakzeptabel werden ließ.

Dies hielt aber junge Autoren nicht davon ab, vor allem die literarischen Zeitschriften mit Prosagedichten und Kurzprosatexten im Übergangsbereich von Vers und Prosa zu beliefern. Da die entsprechenden Redaktionskorrespondenzen meist nicht in gedruckter Form zugänglich sind oder gar als verschollen gelten müssen, lassen sich zum gegenwärtigen Zeitpunkt keine gesicherten Aussagen über die Veröffentlichungspolitik der einzelnen Organe machen, d.h. es bleibt unklar, ob die Herausgeber eher restriktiv oder freizügig beim Abdruck solcher Texte verfuhren. Immerhin ergibt ein Abgleich mit dem Gesamtverzeichnis des deutschsprachigen Schrifttums $(G V)$, daß einige der in Journalen publizierenden Verfasser von Prosagedichten es gar nicht schafften, mit eigenen Buchveröffentlichungen hervorzutreten (Walter Seek, Georg Trepplin), und diejenigen, denen dies gelang, sich durchweg anderer Textsorten bedienten (Heinrich Brömse, Kurt Eisner, Hans Fischer, Ella Lindner, Mite Kremnitz, Karl Röttger, Anna Schapire, Hans Schenk, Toni Schwabe). In beiden Fällen unterblieb ein abermaliger Abdruck der Kurzprosa.

Trotz der insgesamt sich durchaus summierenden Wirkung blieb die literarische Prägekraft der bislang genannten poetae minores für sich genommen relativ gering. Man könnte daher meinen, daß der trivialisierende Einfluß der Epigonen bei der Gattung Prosagedicht letztlich kaum größer war als bei anderen Textsorten. Dem steht freilich der exorbitante Erfolg eines Schriftstellers entgegen, der mit scinen Texten das Verständnis des Genres wahrscheinlich tiefgreifender prägte als alle seine Kollegen: Cäsar Flaischlen (1864-1920). So wenig bekannt dieser Autor heute auch ist, um die Jahrhundertwende gehörte er zu den wichtigen Multiplikatoren im Kulturbetrieb; von 1895 bis 1900 redigierte er etwa dic Zeitschrift Pan. Literaturgeschichtlich zählt Flaischlen zur Gruppe jener Schriftsteller, wdie aus dem Umkreis des Naturalismus zur Heimatliteratur kamen ${ }^{18}$. Nachdem er in den ersten zehn Jah-

- ebenso wie von Schullem - zur wjungösterreichischen Provinzlitteraturu; Hugo Greinz: Die österreichische Provinzlitteratur (= Aus dem Engeren. Litteraturbilder aus deutschen Einzelgauen XII). In: Das litterarische Echo 3 (1900/01), Sp. 884. 
ren seiner Autorschaft ausschließlich Versgedichte - Nachtschatten (1884), Vom Haselnußroi' (1891) - und Dramen - Graf Lothar (1886), Toni Stürmer (1891), Martin Lebnhardt (1895) - verfaßt hatte, wandte er sich mit für einen Epigonen charakteristischer Verspätung der narrativen Prosa - Professor Hardemut. Charakterstudie (1897), Flïgelmüde. Aus dem Leben eines Jeden. Vorstudie zu Jost Syffiedk (1897) - zu. Parallel dazu entdeckte er auch das Prosagedicht als Gestaltungsform für sich. Dessen textuelle Gegensatzstruktur mit ihrer Verkoppelung von गPoesier und Prosa schien sich in idealer Weise als Ausdrucksmedium für die aus Flaischlens Sicht zentralen Antagonismen der Zeit anzubieten: Kultur/Natur, Stadt/Land, Masse/Individuum. Und so betitelte er seinen Band mit "Gedichten in Prosa« programmatisch Von Alltag und Sonne (1898), wobei "Alltag« als Chiffre für die zivilisatorischen Entfremdungstendenzen der Modeme steht ${ }^{19}$ und "Sonne« metonymisch auf die Gesamtheit persönlicher Glückserfahrungen in den Nischenbezirken der Existenz verweist. ${ }^{20}$

Die Texte selbst muten in ihrem Synkretismus wie ein Amalgam aus den einschlägigen Ausprägungstypen des deutschen Prosagedichts der neunziger Jahre an. In ihnen verbindet sich schlafsche Naturidyllik, die gelegentlich wie bei Dauthendey vitalistisch dynamisiert und personifiziert ist, mit Szenen schwülstiger Erotik, wie man sie aus Croissant-Rusts Kurzprosa kennt, allegorischen Phantasien, bei denen Bierbaum Pate gestanden haben könnte, und existentialistischen Parabeln im Stile Turgenevs. ${ }^{21}$ Auch auf der Ebene der formalen Textorganisation lassen sich die entsprechenden Muster wiedererkennen: Ähnlich wie Altenberg ordnet auch Flaischlen seine Prosagedichte zyklisch an, und ebenso wie Bierbaum und Schlaf parzelliert er sie in einer Weise, die es unmöglich macht zu unterscheiden, ob man es mit Abschnitten narrativer Prosa oder freien Rhythmen mit Langzeilen zu tun hat. Um die Verschränkung von Vers und Prosa soweit wie nur möglich voranzutreiben, übernimmt er nicht nur Croissant-Rusts Kleinschreibung des Zeilenanfangs, in einem Fall (»So regnet es sich langsam ein ...4) bedient er sich sogar - abermals Bierbaum und Schlaf folgend - des Reims, um einem Text Kohärenz zu verleihen. Überhaupt spielen in einer ganzen Reihe von Prosagedichten Gestaltungsmittel eine große Rolle, die auf die Lyrik verweisen. So sind nicht nur Wort- und Satzrekurrenzen die be-

19 Stecher bemerkt hierzu: „Und es ist die Großstadt, worin sich dieser unentrinnbare Alltag am dichtesten ballt.« G. [otthilf] Stecher: Cäsar Flaischlen. Kunst und Leben. Stuttgart: Deutsche Verlags-Anstalt 1924, S. 130.

20 Auf die umfassende Verbreitung des Motivs der Sonne in der Literatur der Jahrhundertwende hat besonders Martens hingewiesen: Die Sonne fungiert demnach als mSpenderin der Lebenskraft, Ausgangspunkt und unerschöpfliches Kraftzentrum einer umfassenden Erneuerung des Lebensi; Gunter Martens: Vitalismus und Expressionismus. Ein Beitrag zur Genese und Deutung expressionistischer Stilstrukturen und Motive, S. 90. Liliencron und Croissant-Rust zählten nachweislich »zu den Freunden« Flaischlens; Amanda Schäfer: Anna Croissant-Rust, zum 80. Geburtstag der Dichterin am 10. Dezember. In: Neue Mannheimer Zeitung, 9.12.1940, unpag.; zitiert nach dem Ausschnitt in der Handschriftenabteilung der Stadtbibliothek München (Monacensia), Signatur L 3238. 
vorzugten stilistischen Verfahrensweisen, in vielen Fällen werden diese funktional auch wie ein Refrain eingesetzt, der - gelegentlich geringfügig variiert - den entsprechenden Text entweder rahmt (»Ich muß an das Meer denken ...«, Der Hof, Ecce poeta!, »Das sind so Tage ...«, Nordoststurm) oder ihn in gleichmäßige Abschnitte unterteilt (") Trag Rosen! komm, trag Rosen! ..., Nicht allzu lustig!, "Auch wir werden alt werden ...«, »Ich hab es gerne ...«, »Ganz still einmal ...«, »Mitten in der schönsten Rosenzeit ...«). Es verwundert deshalb kaum, daß die beiden Bandrubriken »Rondos« und »Lieder» explizit auf lyrisch-musikalische Genres verweisen.

Die starke Vorbildwirkung von Vertretern der Münchner Moderne deutet im übrigen darauf hin, daß in Flaischlens Poetik wirkungsästhetische Überlegungen eine erhebliche Rolle spielen. Schon in seinen literarischen Anfängen hat der Autor sich am Modell des Volkslieds orientiert und seine Gedichte bewußt in seinfachsten VersmaBen ${ }^{22}$ gehalten. Er bevorzugte mithin Gestaltungsweisen, die denen artifiziell konstruierter "Kunstpoesie ${ }^{23}$ diametral entgegengesetzt waren. Nur so schien ihm jenes Massenpublikum erreichbar zu sein, an das sich seine Texte adressieren. Stecher bemerkt denn auch zu Recht: »Cäsar Flaischlens Bücher gelten immer und betont rallen und rjeden « $^{24}$. Die Wahl der Textform Prosagedicht macht da keine Ausnahme. Flaischlen interessiert an der Gattung nicht ihr Distinktionspotential, vielmehr begreift er sie als überaus flexiblen Ausdrucksmodus, mit dessen Hilfe die nach wie vor bestehenden sozialen Rezeptionsbarrieren gegenüber sDichtung zu umgehen sind. Der eklektische Rückgriff auf bereits bestehende Muster soll dabei dem Leser ebenso Vertrautheit suggerieren wie der ausgiebige Gebrauch bekannter Topoi und der Einsatz sprachlicher Standardformeln. Der Einsatz von klischierten Gestaltungsmitteln dient also im wesentlichen der Verbreiterung der Adressatenbasis. Zugleich aber soll die typographische Präsentation auch verhindern, daß die Prosagedichte als bloße Wegwerfliteratur behandelt werden. So äußert Flaischlen einmal über seine Texte:

Würde ich fortlaufend drucken - läse man dergleichen, wie man seine Zeinung liest wuppdich - schnuppdich, eins, zwei, drei - und dann will ich, daß die Leute sich beim einzelnen Wort eine etwas intensivere Vorstellung machen möchten, als dies gewöhnlich geschicht ... dazu ist jedes Mittel berechtigt - zumal, wenn ich darauf verzichte, durch absonderliche Stoffe zu reizen. ${ }^{25}$

Bei ihm mutiert das Prosagedicht mithin zu einem volkspädagogischen Erbauungsgenre, das den Eskapismus- und Regressionswünschen breiter Rezipientenschichten entgegenkommt und dessen in handlichen Häppchen verabreichte banale Alltags-

22 G. [otthilf] Stecher: Cäsar Flaischlen. Kunst und Leben, S. 133.

23 Ebd. Da sein »Kunstbegriffu ein Gegenkonzept zum l'art pour l'art markiere, stelle »Cäsar Flaischlen den eigentlichen Antipoden zu Stefan Geonger dar; ebd., S. 88 und 136.

24 Ebd., S. 126.

25 Zitiert nach: ebd., S. 135. 
weisheiten den halbgebildeten Lesern das Gefühl geben, sie würden an der Konsumption von sLiteratur partizipieren.

Aber gerade mit diesem trivialisierten Gattungskonzept hatte Flaischlen ungeheuren Erfolg. Selbiger stellte sich zwar nicht sofort ein, hielt aber um so nachhaltiger an. Während die zweite und dritte Auflage der Prosagedichtsammlung erst 1902 und 1904 erschienen, erlebte Von Alltag und Sonne in der darauffolgenden Dekade durchschnittlich zwei Auflagen von je 1000 gedruckten Exemplaren pro Jahr. Ein signifikanter Sprung ereignet sich dann während des Ersten Weltkriegs: Nachdem allein 1915 immerhin schon 5000 Stück abgesetzt wurden, schnellte die Zahl der verkauften Bücher 1918 auf 26000 hoch. Doch die Verkaufszahlen steigerten sich weiter: 1919 erreichte die Gesamtauflage das 133. Tausend, 1920 war es schon das 180. Tausend und im Jahr darauf das 209. Tausend. Erst danach flaute die Nachfrage wieder ab; 1937 lag der Stand der ausgelieferten Exemplare bei der Marke von $274000 .^{26}$ Das bedeutet, daß Von Alltag und Sonne die mit Abstand erfolgreichste deutschsprachige Prosagedichtsammlung ist, deren Absatzziffern die von Altenbergs populärem Band Wie ich es sebe um ein Vielfaches übertreffen. ${ }^{27}$ Bedenkt man schließlich, daß Flaischlen 1916 unter dem Titel Heimat und Welt eine Auswahl seiner "Gedichte in Vers und Prosa" veröffentlichte, die vier Jahre später schon in 215000 Exemplaren verbreitet war, und sämtliche Prosagedichte von ihm 1921 auch noch einmal in der sechsbändigen Ausgabe seiner Gesammelten Dichtungen abgedruckt wurden, dann kann man ungefähr ermessen, wie nachhaltig die Wirkung seiner Texte auf die Entwicklung der Gattung gewesen sein muß. ${ }^{28}$

Sein Beispiel führt vor, daß Genres nicht nur im Lauf ihrer Entwicklung ihren symbolischen Status verändern, sondern daß ein und dieselbe Ausdrucksform auch zu einem bestimmten Zeitpunkt in ganz unterschiedliche Funktions- und Wertkontexte eingebunden sein kann. Im Hinblick auf den »Prozeß der Ausdifferenzierung von Textsorten hat deshalb Bourdieu zu Recht festgestellt:

In jeder Gattung [...] kommt es tendenziell zu einer Spaltung in einen experimentellen und einen kommerziellen Sektor - zwei Märkte, zwischen denen keine scharf gezogene Grenze angenommen werden darf, die vielmehr nur zwei durch ihre antagonistischen Beziehungen definierte Pole ein und desselben Raums darstellen. ${ }^{29}$

26 Die Angaben lassen sich den verschiedenen Auflagen des Bandes Von Alltag und Sonne entnehmen, denn der Verlag druckte diese Informationen zu Werbezwecken regelmäßig mit ab. Von Wie ich es sehe erschien 1928 die 19. und 20. Auflage; vgl. Peter Altenberg: Gesammelte Werke in fünf Bänden, Bd. 1, S. 383, bzw. Bd. 2, S. 477. Die Gesamtauflage von Altenbergs Werken erreichte dem Klappentext der Edition zufolge im genannten Jahr $\$ 108000$ Exemplarew.

Damit bleiben die Absatzzahlen von Flaischlens Prosagedichten nicht allzu weit hinter dem Ausnahmeerfolg von Victor von Scheffels Trompeter von Säckingen (1854) zurück, dem mit Abstand meistverkauften Versband im späten 19. und frühen 20. Jahrhundert; vgl. die Angaben bei Eva D. Becker: Literaturverbreitung, S. 134. 
Diese Spaltung nun ist im Bereich des Genres Prosagedicht spätestens mit Flaischlens Texten erreicht, wie überhaupt das - von Rilke in seinem Vortrag Moderme Lyrik bereits konstatierte - Überhandnehmen epigonaler Autoren in der zweiten Hälfte der neunziger Jahre indiziert, daß um 1900 die Gattungsentwicklung ein neues Stadium erreicht. Fortan gibt es nicht nur einen populären und einen elitären Sektor des Textmusters, das Spektrum infrage kommender Bezugstexte hat sich auch fast bis zur Unüberschaubarkeit vergrößert: Neben die moralistischen Allegorien Turgenevs, ${ }^{30}$ Liliencrons Vorstöße zur Prosaisierung der Poesie, die Textstatusexperimente von Bierbaum und Croissant-Rust, Dauthendeys und Schlafs Versuche einer Engführung von Naturwissenschaft und Dichtungstheorie sowie Altenbergs an Huysmans angelehnte "Ausspartechnik» im Dienst ästhetischer Entdifferenzierung sind die rhetorisierten Texte Nietzsches, die Langzeilendichtung Whitmans und die gesamte Tradition der spoetischen Prosac von Jean Paul bis hin zu den Psalmen der Bibel getreten. Dazu kommen noch zahlreiche weitere Spielarten kurzer Prosa von Autoren aus verschiedenen Ländern Europas: Sigbjörn Obstfelder, ${ }^{31}$ Franz und Émile Erens, ${ }^{32}$ Arne Garborg, Gaston le Poil, William Novalis. ${ }^{33}$

Es fällt freilich auf, daß die gattungsbegründenden französischen Muster des poème en prose in diesem Zusammenhang nur eine marginale Rolle spielen. Das hängt in erster Linie mit dem Fehlen von Übersetzungen zusammen. ${ }^{34}$ Eine erste

30 Ein frühes Beispiel für einen deutschen Autor, der sich in erster Linie an dem von Schopenhauer beeinflußten Gestus desillusionierter Selbstbefragung der Gedichte in Prosa Turgenevs, nicht aber an anderen zur Verfügung stehenden Gattungsvorbildem (wie etwa Liliencrons kurzer Prosa) orientiert, ist Otto Kemmer. Seine Textsammlung Gedichte in Prasa. Aus den Papieren eines Pessimisten (1885) kann im übrigen als frühester Prosagedichtband in deutscher Sprache angesehen werden.

31 Vgl. Sigbjörn Obstfelder: Pilgerfahrten. Aus dem Nachlaß des Dichters. Deutsch von Luise Wolf. Stuttgart: Axel Juncker 1905.

32 Vgl. die Texte Kirmess und Eine Ekstase von Émile Erens sowie Kaiser-Einqug in Amslerdam von Franz Erens, die in Deutschland als Beispiele wfür die Prosalyrik der jüngeren holländischen Poeten « präsentiert werden; Proben junghollaendischer Poesie. Uebertragen von Émile Erens. In: Neue deutsche Rundschau 5 (1894), S. 57

33 Vgl. Kleine Gedichte in Prosa (Garborg - Le Poil - Novalis). In: Ebd., S. $496-498$ (enthält die Texte Verzueiflungen von Ame Garborg, Die traurige Trösterin von Gaston le Poil und Das Mädchen und die Lilien von William Novalis).

34

Bei Baudelaire absorbierte der in den Fleurs du mal nachgerade spektakulär inszenierte Kontrast von schockierendem Inhalt und strenger Textform lange Zeit die gesamte Aufmerksamkeit, und im Falle Rimbauds und Mallarmés mkonzentrieren sich die Bemühungen der deutschen Nachdichter der Jahrhundertwende auf das Frühwerk, in dem die traditionellen metrischen Formen noch nicht gesprengt und die romantischen Nachllänge unüberhörbar sind«; Manfred Gsteiger. Französische Symbolisten in der deutschen Literatur der Jahrhundertwende (1869-1914), S. 214f. Ein kaum bekanntes frühes Beispiel einer Teilübersetzung der Petits poimes en prose stellen die zu Lebzeiten unveröffentlichten Übertragungen Peter Hilles (Bestimmung, Die Gaben des Mondes) dar. Vgl. hierzu Wolfgang Bunzel: "Echte Lyrik nährt sich von der feinsten Epik.» Peter Hilles Kurzprosa im ästhetischen Kontext ihrer 
deutsche Gesamtübertragung der Petit poèmes en prose war nicht vor 1902 zugänglich, ${ }^{35}$ Rimbauds Illuminations erschienen in Auswahl erstmals $1907,{ }^{36}$ und Mallarmés Prosagedichte blieben sogar bis zum Ende des expressionistischen Jahrzehnts unübersetzt. ${ }^{37}$ Die wenigen zuvor in Zeitschriften publizierten Übersetzungen einzelner Texte ${ }^{38}$ vermögen an diesem Befund nicht wirklich etwas zu ändern, zumal sie

Zeit. Einen aufschlußreichen Hinweis auf das Gattungsverständnis Hilles gibt dabei der Umstand, daß der Autor Baudelaires Textvorlage als "Lieder in Prasow bezeichnet hat; Walther Pfannmüller: Der Nachlaß Peter Hilles. Gotha. o.V. 1940, S. 85. Vgl. hierzu auch Gertrud Weigert: Peter Hille. Untersuchungen und Texte. Königsberg. Gräfe und Unzer 1931 (= Königsberger Deutsche Forschungen 9), S. 73f.

Charles Baudelaire: Gedichte in Vers und Prosa. Übersetzt von Camill Hoffmann und Stefan Zweig. Leipzig: Hermann Seemann Nachfolger 1902.

Arthur Rimbaud. Leben und Dichtung. Übertragen von K.L. Ammer [= Karl Klammer]. Eingeleitet von Stefan Zweig. Leipzig. Insel 1907. (Enthält 14 von 42 Texten der Illuminations.) Krüger konstatiert in diesem Zusammenhang zu Reche $" D i e$ eigentliche Rezeption Rimbauds in Deutschland beginnt mit der 1907 im Insel-Verlag erschienenen Übertragung Karl Klammers. Eva Krüger. Todesphantasien. Georg Heyms Rezeption der Lyrik Baudelaires und Rimbauds. Frankfurt a.M./Berlin/Bem/New York/Paris/Wien: Lang 1993 (= Berliner Beiträge zur neueren deutschen Literaturgeschichte 18), S. 25. Als weitere Übersetzungen wären zu nennen: A. R.: Erleuchtungen. Gedichte in Prosa. Deutsche Nachdichtung von Paul Zech. Leipzig. Wolkenwanderer-Verlag 1924 (= Der Schatzbehalter 5); Das gesammelte Werk des Jean-Arthur Rimbaud. In freier deutscher Nachdichtung von Paul Zech. Leipzig: Wolkenwanderer-Verlag 1927; A. R.: Leben, Werk, Briefe. Übertragen und hrsg. von Alfred Wolfenstein. Berlin: Internationale Bibliothek o.J. [1930]. Vgl. in diesem Kontext auch Manfred Gsteiger: Rimbaud en allemand: Klammer, Zech, Wolfenstein. In: Proceedings of the IXth Congress of the International Comparative Literature Association / Actes du IXe congrès de l'Association intemationale de littérature comparée, Innsbruck 1979. Bd. 2: Literary Communication and Reception / Communication littéraire et réception / Literarische Kommunikation und Rezeption. Ed. by / Réd. par Zoran Konstantinović, Manfred Naumann, Hans Robert Jauß. Technische Redaktion: Ingrid Vama. Innsbruck Verlag des Instituts für Sprachwissenschaft der Universität Innsbruck 1980 (= Innsbrucker Beiträge zur Kulturwissenschaft. Sonderheft 46), S. 377-383.

37 Stéphane Mallarmé: Der Nachmittag eines Fauns und einige Blätter Prosa. Die Übertragung in der Auswahl von Artur Roessler besorgte Erwin Rieger. Wien: Avalun-Verlag o.J. [1920] (= Avalun-Druck 5).

Vgl. Charles Baudelaire: Gedichte in Prosa [übersetzt von Felix Dörmann]. In: Modeme Rundschau (Wien) 3 (1890), S. 447-449 (enthält Der Fremdling, Die Verzaeiflung der Alien, Mondlichtgaben, Berauschet Euch); C. B.: Gedichte in Prosa [übersetzt von Robert Fischer]. In: Moderne Rundschau (Wien) 4 (1891), S. 65f. (Der Narr und die Venus, Comfiteor des Künstlers); C. B.: Der Fremdling [übersetzt von Georg Bachmann]. In: Die Gesellschaft 14 (1898), S. 185f.; C. B.: Glaubensbekenntnis eines Künstlers [übersetzt von Ella Werner]. In: Die Gesellschaft $16(1900)$, Bd. 1, S. 167f.; C. B.: Gedichte in Prosa [übersetzt von Ella Werner]. In: Die Gesellschaft 16 (1900), Bd. 3, S. 108-110 (enthält Der Fnemdling, Die Verpweif lung der Greisin, Die Fenster und Ein Spöster); Arthur Rimbaud: Nach der Sündflut. Aus dem Französischen von E.R. Weiss. In: Wiener Rundschau 5 (1901), S. 97. Siehe hierzu die bibliographischen Angaben bei Manfred Gsteiger: Französische Symbolisten in der deutschen Literatur der Jahrhundertwende (1869-1914). 
gelegentlich nur das Unverständnis belegen, das der Textsorte Prosagedicht in Deutschland entgegengebracht wurde. ${ }^{39}$ So verwandelte etwa Georg Bachmann Baudelaires in Dialogform abgefaßten Text L'Étranger - der allerersten Übertragung eines Petit poème en prose ins Deutsche - in ein stichomythisches Versgedicht. ${ }^{40}$ Die nachmals berühmten französischen Ausprägungen des Genres waren also nur jenen Autoren zugänglich, die sich entweder eine zeitlang im Nachbarland aufhielten (George, Rilke) oder zumindest über gute Sprachkenntnisse verfügten und sich obendrein durch eine ausgeprägte internationale Rezeptivität auszeichneten (Hofmannsthal, Altenberg).

Faktisch verläuft damit in der Entwicklungsgeschichte des deutschen Prosagedichts eine Trennlinie zwischen Schriftstellern, die mit französischen Gattungmustern vertraut waren, und solchen, die sie nicht kannten. Daß dieser Riß jedoch kaum auffallt, liegt daran, daß Hofmannsthal seine Prosagedichte nicht veröffentlichte, George bei seiner Kurzprosa auf eine Zuweisung zu diesem Genre verzichtete und Rilke seine Entscheidung für die Prosaform nach der Publikation der entsprechenden Texte wieder revozierte. So bleibt einzig und allein Altenberg als gewichtiger Vertreter der ersten Fraktion übrig; allerdings wurde er in der öffentlichen Wahrnehmung so stark mit dem kulturellen Umfeld Österreich identifiziert, daß seine Bindung an französische Vorbilder aus dem Blick geriet. Innerhalb der deutschen Gattungsentwicklung dominiert daher die Orientierung an nichtfranzösischen Textmodellen außerhalb der Tradition des poème en prose. Verstärkt wurde diese Tendenz noch durch die Vertextungspraxis der poetae minores, die in ihrer Fixierung auf deutsche Vorbilder nur einen sehr schmalen Ausschnitt aus der Gesamtheit der Gestaltungsmöglichkeiten nutzten. Anstatt die eigene dichterische Produktion in den Kontext der internationalen Literaturentwicklung zu stellen, beschränkten sich diese Autoren bei der Wahl ihrer Referenztexte durchweg auf das vergleichsweise enge Feld der eigenen kulturellen Tradition. Unkenntnis oder Ignoranz gegenüber fremdkulturellen Innovationen aber bewirken zwangsläufig einen Entwicklungsrückstand, der allenfalls durch besonders kreativen Umgang mit dem zur Verfügung stehenden Ausdrucksrepertoire des nationalen Literatursystems wettgemacht werden kann. Bleibt ein solcher jedoch aus, ermangelt den Texten meist jenes Originalitäts-

39 Die Tatsache, ndaß die Prosagedichte爪 Baudelaires, Rimbauds und Mallarmés "verhältnismäßig selten verdeutscht wurden «, ist - anders als Gsteiger meint - wohl doch nicht so sehr ein "Zeichen ihrer eigentlichen Unübersetzbarkeitu als vielmehr "Ausdruck einer verbreiteten Ablehnung oder doch eines Widerstandes gegen die neuen Formen«; ebd., S. 215 .

$40 \mathrm{Da}$ die Tendenz, das poème en prose als eine Art ’prosaischesı Versgedicht aufzufassen, um 1900 weitverbreitet war und sich auch in der übersetzerischen Praxis niedergeschlagen hat, belegt etwa die Umformung dreier Prosagedichte Maeterlincks in freie Rhythmen; vgl. Maurice Maeterlinck: Gedichte in Prosa. [Verdeutscht von Karl Lothar Ammer.] In: Wiener Rundschau 3 (1898/99), S. 537-540. 
potential, das es erlauben würde, ihren Verfassern einen Status außerhalb der Schar der bloßen Nachahmer zuzuerkennen.

Das Überhandnehmen der Epigonen und die in Reaktion darauf sich verstärkenden formkonservativen Tendenzen ${ }^{41}$ in der Literatur hatten schließlich zur Folge, daß das Genre Prosagedicht nach 1900 in eine ernste Krise geriet. Indiz dafür ist der merkliche Rückgang publizierter Kurzprosa: Nicht nur in den Journalen nahm die Zahl entsprechender Texte deutlich ab, im ersten Jahrzehnt des 20 . Jahrhunderts erschien auch kein einziger Sammelband mehr, der ausschließlich Prosagedichte enthält. Damit gelangte der Evolutionsprozeß der Gattung an den Punkt, an dem das Textmodell integraler Bestandteil des Kanons möglicher Gestaltungsweisen geworden ist. Allerdings unterblieb jene nachträgliche theoretische Legitimierung durch die akademische Poetik, die bis dahin immer nötig war, damit eine Ausdrucksform soffiziellec Anerkennung fand. Diese verweigerte Akzeptanz nun sicherte dem Prosagedicht weiterhin eine exzentrische Position im Gattungsgefüge und schuf so die Voraussetzung für seine erneute Refunktionalisierung im Expressionismus. ${ }^{42}$

Während die im Sektor eingeschränkter Produktion operierenden expressionistischen Autoren das Genre durchweg als Spielart der Lyrik begriffen, gab es bei den Epigonen im zweiten Jahrzehnt des 20. Jahrhunderts mehrfach Versuche, durch gezielte Reprisen die nichtlyrische Gattungstradition zu stärken. Als wohl bedeutendster Vertreter dieses Texttyps kann Armin T. Wegner (1886-1976) gelten, ${ }^{43}$ der 1910 mit einem Band Gedichte in Prosa hervortrat. ${ }^{44}$ Wie schon der Untertitel "Ein Skizzenbuch aus Heimat und Wanderschaft«, mehr aber noch die Subsumierung der Texte unter Rubriken ("Stimmungen, Liebesgedichte in Prosa", "Skizzen und Aquarelle", "Lehrgedichte in Prosa", "Märchen und Saytren«, "Balladen in Prosa», "Grotesken und Allegorien () zeigt, schließt der Autor direkt an die naturalistische Tradition narrativer Kurzformen an. Es verwundert denn auch kaum, wenn sich hier neben kurzen Prosagedichten mit einem Umfang von lediglich 40 oder 50 Zeilen diverse län-

41 Ihren deutlichsten Ausdruck hat diese Tendenz in Paul Emsts Schrift Der Weg zur Form. Ästhetische Abhandlungen, tornebmlich qur Tragödie und Novelle (1906) gefunden. Vgl. hierzu Andreas Wöhrmann: Das Programm der Neuklassik. Die Konzeption einer modemen Tragödie bei Paul Emst, Wilhelm von Scholz und Samuel Lublinski. Frankfurt a.M.: Lang 1979 (= Europäische Hochschulschriften I/301). Siehe Kapitel III/9.

43 Vgl. Reinhard M.G. Nickisch: A.T. Wegner. Wuppertal: Hammer 1982, Johanna WemickeRothmayer: Armin Theophil Wegner. Gesellschaftserfahrung und literarisches Werk. Frankfurt a.M./Bern: Lang 1982 (= Europäische Hochschulschriften I/503) und Martin Rooney: Leben und Werk Armin T. Wegners (1886-1976) im Kontext der sozio-politischen und kulturellen Entwicklungen in Deutschland. Frankfurt a.M.: Haag + Herchen 1984. Wegner. Eine Auswahlbibliographie bearbeitet von Hedwig Bieber. Als Arbeitsmanuskript veröffentlicht. Dortmund: Stadtbücherei Dortmund 1971 (= Dichter und Denker unserer Zeit 38). 
gere Erzähltexte finden, die sich in einzelnen Fällen sogar über 16 (Das Bett) oder auch 17 Druckseiten (Der Mäblenstein) erstrecken. Die Unterschiede zwischen den einzelnen Subgenres sind jedoch gering. In fast allen Texten des Bandes geht es um die allegorische Konfigurierung von Situationen aus dem Bereich individueller Lebensführung oder sozialer Interaktion, die im Sinne moderner Moralistik zu erkenntnisfördernder Reflexion einladen soll. Hin und wieder freilich wird die lebenspraktische Anwendung dieser Denkanstöße bereits soweit vorweggenommen, daß die Texte zu literarisch vermittelten Handlungsanleitungen zu degenierieren drohen.

Als Muster für diesen Aussagemodus fungieren in erster Linie Turgenevs Stichotvorenija v proze und Baudelaires Petits poèmes en prose, die Wegner vermutlich beide in neueren Übersetzungen rezipiert hat. ${ }^{45}$ Manche seiner Texte sind den Prosagedichten der Genannten in Aufbau und Struktur so ähnlich, daß sie als regelrechte Variationen angesehen werden können: Der Turm etwa rekonstelliert Turgenes Rargovor (Eine Unterhaltung), Die Bank der alten Leute greift auf Baudelaires Les Veuves zurück, und Die Greisin mutet wie eine Verkreuzung von Baudelaires Chacun sa chimère mit Turgenevs Starucha (Die Alte bzw. Die alte Frau) an. Daneben verweisen aber auch Themen wie die menschliche Projektion eigener Wünsche auf die Natur (Der KünstLer), die dünkelhafte Ablehnung alles Neuen (Ein Philister) oder Undankbarkeit gegenüber Wohlmeinenden (Es ist eine weite Kluft, Ein Prophet), Motive wie sprechende Gegenstände (Der Apfelbaum, Der Müblenstein) oder die Verbindung von Tier und Kot (Der Künstler) sowie die darstellerische Bevorzugung von Deklassierten, Alten und Außenseiterfiguren (Die Orange, Die Großmutter, Die Bank der alten Leute, Die Rose, Aus der Schulzeit, Das Fenster, Die Seeigel-Grotte, Kornfeld, Der Schiffbrüchige) ${ }^{46}$ bzw. die Gestaltung von Träumen (Die Kirche, Die drei Träume) und Visionen direkt auf die

45 Nachdem die älteren Ausgaben der Stichotvorenija v prož, darunter auch die wohifeile in Reclams Universal-Bibliothek, längst vergriffen waren, erschien Anfang des 20. Jahrhunderts eine sehr ansprechend aufgemachte Neuübersetzung mit Buchschmuck von Heinrich Vogeler; vgl. Iwan Turgenjeff: Gedichte in Prosa. Deutsch von Th. Commichau. Leipzig: Insel o.J. [1903], ${ }^{2}$ [1910]. Die Petits poemes en prose waren gleich mehrfach in deutschen Übertragungen greifbar; neben dem Band von Hoffmann und Zweig (1902) wären hier zu nennen: Charles Baudelaire's Werke in deutscher Ausgabe. Hrsg. von Max Bruns. Bd. 1: Novellen und kleine Dichtungen in Prosa. Übersetzt von Margarete Bruns. Minden: J.C.C. Bruns 1904, sowie: Charles Baudelaire: Gedichte und Skizzen. Hrsg. und eingeleitet von Fritz Gundlach. Leipzig. Reclam 1908 (= Reclams Universal-Bibliothek 5076). Die Bezeichnung der "Kleinen Gedichte in Prosar als "Skizzen in Gundlachs Übersetzung legt nahe, daß Wegner diese Ausgabe benutzt hat. Wenn dem tatsächlich so ist, dann wäre das ein weiterer Beleg für die starke Prägewirkung von Translationen.

46 Dies geht bis hin zur Charakteristik einzelner Personen. Wenn es beispielsweise von einem Schiffbrüchigen heißt, wer hatte weder Weib noch Kind, noch nannte er Eltern sein eigen; er glaubte nicht an Gott, noch hatte er Freunde oder ein Vaterlandk, dann ruft Wegner mit dieser Beschreibung fast wörtlich die zentralen Merkmale der Figur des Fremden in Baudelaires Prosagedicht L'Étranger auf; Armin T. [heophil] Wegner: Gedichte in Prosa. Ein Skizzenbuch aus Heimat und Wanderschaft. Berlin: Egon Fleischel 1910, S. 171. 
Vorbilder. Die Art der Bezugnahme auf die Prätexte deutet dabei darauf hin, daß Wegner ihnen weitgehend distanzlos begegnete. So nimmt er beispielsweise die zuweilen bis zum Zynismus gehende illusionsbrechende Ironie der Petits poèmes en prose in keiner Weise wahr. Anders als bei Turgenev sind seine Prosagedichte denn auch nicht als korrigierende Umschriften anzusehen, vielmehr orientiert sich der Autor an den Vorbildern wie an nachzuahmenden Modelltexten.

Ganz ähnlich gestaltet auch Walter Georgi (1871-1924) in seinen Gedichten in Prosa (1920) allegorische Situationen, die teilweise im menschlichen Lebensalltag, teilweise aber auch in irrealen Szenerien angesiedelt sind. Der Umstand, daß darin sogar göttliche Instanzen als Akteure begegnen (Die blaue Blume, Die Geburt der Atbene, Der Herngott und die Tannen, Der Brief vom lieben Goth) verleiht manchen der Texte märchenhafte Züge, ${ }^{47}$ rückt sie aber vor allem in die Nähe gleichnishafter Erbaulichkeit. Wie in den phantastischen Prosagedichten geht es auch in jenen, die Träume oder Visionen schildern (Ein Traum, "Ihr lacht vielleicht ...", Halluzination), darum, das wahrnehmende Subjekt zum Innehalten zu veranlassen. Die so entstehende momenthafte Distanz zum Geschehen setzt dann Reflexionen in Gang, die anschließend bei Bedarf noch sentenzenhaft zusammengefaßt werden können. Referenzpunkt sind hier eindeutig Turgenevs Stichotvorenija $v$ proze, wie sich vielleicht am deutlichsten an der Rekonstellierung des Blicktauschs zwischen Mensch und Tier aus Sobaka (Der Hund) in dem Text Ein Traum zeigt.

Während Wegner und Georgi in ihren besten Prosagedichten immerhin das Niveau der Stichotvorenija v proze erreichen, gleiten dagegen Paula von Bülow-Lindens (1833-1920) Versuche in diesem Genre zur Gänze in den Bereich des Trivialen ab. Der eigenartige Titel ihrer Sammlung, Plaudereien und Gedicbte in Prosa (1913), die sie unter dem Pseudonym G. von der Elda publizierte, offenbart bereits in hinreichender Deutlichkeit, daß hier das Textmodell in den Einzugsbereich anspruchsloser Gebrauchsliteratur gerät. Und tatsächlich überschreitet die Fülle von entleerten Redeschablonen und sprachlichen Gemeinplätzen, die sich in Bülow-Lindens Kurzprosa finden, jede ästhetische Peinlichkeitsschwelle. Allerweltsweisheiten werden bedenkenlos zu maximenartigen Merksprüchen komprimiert, die überdies häufig Anleihen bei igeflügelten، Worten ()Glücklich ist, wer vergißt, / was nicht mehr zu ändern ist. $\left.{ }^{48}\right)$ nehmen. Die Autorin verquickt in ihren Texten die bei Flaischlen begegnende bürgerliche Dichotomisierung von "Alltag« und Lebensemphase mit Altenbergs erzieherischem Anspruch (»Rüttle dich auf und werdel / [...] - werde Mensch. ${ }^{49}$ ) und Turgenevs allegorischer Aphoristik zu einer hochideologischen, weil in den Bereich

47 Der letzte Text der Sammlung trägt nicht zufällig die Überschrift Das Märchen einer jungen Tanne; vgl. Walter Georgi: Gedichte in Prosa. Berlin: Axel Juncker o.J. [1920], S. 63-65. O.W. Barth 1913, S. 34. 
bürgerlicher Erbaulichkeit getriebenen Mixtur angewandter Lebenshilfe. ${ }^{50}$ Sie propagiert dabei ungeniert eine Haltung im Umgang mit den sozialen und kulturellen $\mathrm{Be}$ dingungen der menschlichen Existenz, die faktisch resignativ ist, sich aber als heiteres Einverständnis mit dem scheinbar Unabänderlichen gibt. Die Prosaform fungiert in diesem Zusammenhang als vertrauter, Rezeptionsbarrieren senkender Gestaltungsmodus, der aus den kurzen bis sehr kurzen Texten leicht zu konsumierende Lektürehäppchen mit Tröstungsfunktion macht.

Von ähnlich sentenzenhafter Trivialität wie Bülow-Lindens Band ist auch Rudolf Alberts (*1899) mSkizzenbuch schlechterkomplementäre Gegenstück zu den Plaudereien darstellt: Anstelle der häuslichen Freuden weiblicher Entsagung werden hier männlicher Kampfesmut, "Selbstzucht und Willenskraft" ${ }^{51}$ gepriesen.

Wie sehr sich das Gattungsmodell Prosagedicht zu Beginn des 20. Jahrhunderts mit - zumindest vom Umfang her - benachbarten narrativen Textsorten amalgamierte, zeigen besonders deutlich die 1920 unter dem Gesamttitel Gedichte in Prosa in insgesamt drei Bänden erschienenen Kurzprosastücke des beliebten Essayisten und Unterhaltungsschriftstellers Alexander von Gleichen-Rußwurm (1865-1947). Die Tatsache, daß der erste Teil der Trilogie, Der Narrenturm, schon fünf Jahre früher separat veröffentlicht worden ist, damals aber nur den - bei der Zweitpublikation beibehaltenen - Untertitel »Grotesken und Satiren« getragen hat, verdeutlicht, wie sehr die Gattungsbezeichnung Prosagedicht zu einem fast beliebig einsetzbaren Etikett geworden ist, deren Anwendung zunehmend weniger im Dienst auktorialer Poetik steht, sondern sich vorrangig am buchhändlerischen Ideal der Marktgängigkeit ausrichtet. ${ }^{52}$ Und so bietet sich auch Gleichen-Rußwurms Publikationszyklus mit den

50 Selbst Anklänge an die Petits poìmes en prose lassen sich erkennen. So greift etwa der Anfang von Miserere auf den Beginn von Baudelaires Prosagedicht $L$ Confiteor de l'artiste zurück: "Betrachte die stillen Herbsttage, wie sie so eindringlich sind, / Wie ihre sterbende Schönheit des [recte: das] Gemüt bewegt - es in schmerzliche Schwingungen bringt - - - «; ebd., S. 57. Rudolf Albert: Sonntagsträume. Ein Skizzenbuch. Dresden: Strom-Verlag o.]. [1918], S. 31. In einem anderen Text heißt es: "Nur wer seinen Mann stellt im Sturm der Zeiten, kennt die Seligkeit heiliger Feierabendstunden und die Feststimmung innerer Zufriedenheit.. Ebd., S. 51. Eine zeitgeschichtlich beklemmende Pointe erhält dieses Bekenntnis zu maskulinem Heroismus durch den Umstand, daß Alberts Texte - wie die Angabe "Beauraing (Belgien) 1918x im Geleitwort nahelegt - offenbar im Zusammenhang mit einem Fronteinsatz im Ersten Weltkrieg entstanden sind.

$\mathrm{Vgl}$. in diesem Zusammenhang auch Zweigs Verdikt: "Die Skizze ist - außer bei den geborenen Kleinkünstlem - fast nie Innentrieb und Selbstzweck, denn ihr Gravitationspunkt liegt in der Unterhaltsamkeit; selten ist sie mehr als eine flüchtige Kraft, die die Aufmerksamkeit für Minuten in eigengeschaffene Welten führt, die aber mit der letzten Zeile wieder in sich zerrinnen. So kann uns eine einzelne Skizze nie den ganzen Künstler offenbaren; erst eine Sammlung giebt mit dem Ineinandergreifen der Ergänzungen und Korrekturen ein einheitliches Bild seiner Persönlichkeit. Darum ist die Zusammenfassung cinzelner Skizzen zu einem Bande immer eine Prätension und gleichbedeutend mit dem Verlangen, das äu- 
weiteren Teilbänden Die Traumbrücke und Die schwere Krone insgesamt gesehen als reichlich beliebiges compositum mixtum verschiedenster Spielarten kürzerer Prosa dar. Zwar finden sich selbst hier noch vereinzelte Bezüge auf die Genrevorbilder Baudelaire und Turgenev - so greift etwa Drei Spieler und drei Teufel Ein Nachtspuk Motive aus den Petits poèmes en prose auf (zu nennen wären hier Portraits de mattresses und $L$ Joueur généreux) -, doch weist das Vorhandensein mehrerer umfangreicherer Texte überdeutlich darauf hin, daß hier faktisch ganz verschiedenartige Typen narrativer Prosa miteinander kombiniert wurden.

Das Prosagedicht büßte also nach der Jahrhundertwende sein Gattungsprofil weitgehend ein. Nach wie vor aber bestand durch die Existenz einer Vielzahl periodischer Publikationsorgane ein enormer Bedarf nach kurzen, rasch rezipierbaren Prosatexten, der befriedigt werden wollte. Bereits existierende Textsorten wurden daher revitalisiert, neue bildeten sich heraus, wobei sich besonders der Übergangsbereich zwischen Fiktion und Nichtfiktion als geeigneter Nährboden für die Schaffung bislang unerprobter Gestaltungsmuster erwies. Schon bald nach 1900 gab es eine nahezu unüberschaubare Vielzahl kurzer Prosagenres. Und so entwickelte sich wdie Kurzprosa [...] im 20. Jahrhundert zu einer der fruchtbarsten Gattungen der literarischen Moderne ${ }^{53}$ insgesamt. Freilich handelt es sich nicht mehr um einen homogenen Texttyp, vielmehr deckt der Begriff Kleine Prosar nun ein sbreit gefächertes Gattungsfeld « ${ }^{54}$ ab, das von der Anekdote ${ }^{55}$ über die Glosse ${ }^{56}$ und die Aufzeichnung ${ }^{57}$ bis hin zum Denkbild ${ }^{58}$ und zur short story ${ }^{59}$ reicht, vielfach auch auf

Berlich Zusammenhanglose unter dem Gesichtswinkel einer inneren Einheit und Notwendigkeit betrachtet zu sehen [...] [;] auf keinem Gebiete schöpferischer Bethätigung ist der Dilettantismus und die leichtfertige Buchmacherei häufiger, nirgends die Sucht größer, journalistischen Eintagsfliegen künstliche Lebensdauer zu verleihen, materielle Motive in künstlerische umdeuten zu wollen.« Stefan Zweig. Skizzen- und Novellenbücher. In: Das litterarische Echo 5 (1902/03), Sp. 744.

53 Dirk Göttsche: Denkbilder der Zeitgenossenschaft. Entwicklungen modemer Kurzprosa bei Marie Luise Kaschnitz. In: D. G. (Hrsg.): „Für eine aufmerksamere und nachdenklichete Weltw. Beiträge zu Marie Luise Kaschnitz. Stuttgart/Weimar: Metzler 2001, S. 80.

54 Ebd., S. 82. Siehe hierzu generell Viktor Żmegac: Klein- und Kleinstformen der Dichtung. In: Aleksander Flaker/V. Ż. (Hrsg.): Formalismus, Strukturalismus und Geschichte. Zur Literaturtheorie und Methodologie in der Sowjetunion, CSSR, Polen und Jugoslawien, S. 131-144.

55 Vgl. Volker Weber: Anekdote. Die andere Geschichte. Erscheinungsformen der Anekdote in der deutschen Literatur, Geschichtsschreibung und Philosophie. Tübingen: Stauffenburg 1993 (= Stauffenburg Colloquium 26).

56 Kersten beispielsweise erklärt kurzerhand: »Die einzig mögliche Ausdrucksform ist für uns das Gedicht und die Glosse. Alles andere ist Produktionsaffigkeitus Hugo Kersten: Leo Matthias. Der jüngste Tag. In: Die Aktion 4 (1913/14), Nr. 21, Sp. 466. Vgl. Ernst Rohmer: Die literarische Glosse. Untersuchungen zu Begriffsgeschichte, Funktion und Literarizität einer Textsorte. Erlangen: Palm \& Enke 1988 (= Erlanger Studien 79).

57 Vgl. Thomas Lappe: Die Aufzeichnung. Typologie einer literarischen Kurzform im 20. Jahr- 
die Aphoristik ${ }^{60}$ ausgreift. Das Prosagedicht indes verschwand nicht einfach in diesem Kontinuum, sondern erhielt sich - nicht zuletzt wegen der von ihm mittlerweile ausgebildeten Vertextungstraditionen - als Genremodell, auf das bei Bedarf jederzeit zurückgegriffen werden konnte. ${ }^{61}$

hundert. Aachen: Alano/Rader-Publ. 1991. Georg Kulka etwa nennt seine Kurzprosatexte, die er z.T. zusammen mit Verslyrik veröffentlicht, "Aufzeichnung(en)» oder maufzeichnende Prosa«. Er folgt mit dieser Namengebung der redaktionellen Praxis der Zeitschrift Die Dichtung, in der die präsentierten Texte in vier unterschiedlichen Rubriken dargeboten wurden: "Die Epik», "Die Lyrik» »Die Dramatik» und "Die Aufzeichnungw. Vgl. in diesem Zusammenhang außer Kulkas Buch Der Stiefbruder. Aufzichnung und Lyrik (1920) die Bände Auch ich, auch du. Aufzichnungen eines Imen (1919) von Hans Siemsen und Adelina ader Der Abschied vom neunzebnten Lebensjabr. Aufzeichnungen (1920) von Bohuslav Kokoschka. Zu Kulkas Poetik siehe den von Helmut Kreuzer in Zusammenarbeit mit Reinhard Döhl verfaßten Aufsatz: Georg Kulka und Jean Paul. Ein Hinweis auf expressionistische Centonen. In: Deutsche Vierteljahrsschrift für Literaturwissenschaft und Geistesgeschichte 40 (1966), S. 567 576, und Gerhard Sauders »Nachwort« in: Georg Kulka: Werke. Hrsg. von G. S. unter Mitarbeit von Reiner Wild und Eckhard Faul. München: edition text + kritik 1987 (= Frühe Texte zur Moderne), S. 289-330.

Vgl. Eberhard Wilhelm Schulz: Zum Wort „Denkbild火. In: E.W. S.: Wort und Zeit. Aufsätze und Vorträge zur Literaturgeschichte. Neumünster: Wachholtz 1968 (= Kieler Studien zur deutschen Literaturgeschichte 6), S. 218-252; Heinz Schlaffer: Denkbilder. Eine kleine Prosaform zwischen Dichtung und Gesellschaftstheorie. In: Wolfgang Kuttenkeuler (Hrsg.): Poesie und Politik. Zur Situation der Literatur in Deutschland. Stuttgart/Berlin/Köln/ Mainz: Kohlhammer 1973 (= Sprache und Literatur 73), S. 137-154; Dirk Göttsche: Denkbilder der Zeitgenossenschaft. Entwicklungen modemer Kurzprosa bei Marie Luise Kaschnitz. Siehe aber auch Helmut Amtzen: Philosophic als Literatur: Kurze Prosa von Lichtenberg bis Bloch. In: Probleme der Moderne. Studien zur deutschen Literatur von Nietzsche bis Brecht. Festschrift für Walter Sokel. Hrsg. von Benjamin Bennett, Anton Kaes, William J. Lillyman. Tübingen: Niemeyer 1983, S. 51-66, und Michael Esders: Begriffs-Gesten. Philosophie als kurze Prosa von Friedrich Schlegel bis Adorno. Frankfurt a.M./Berlin/ Bern/Bruxelles/New York/Oxford/Wien: Lang 2000 (= Literatur als Sprache 14).

Aus der ausufemden Forschungsliteratur zur short story sei hier - wegen des Bezugs zum Gattungsmodell Prosagedicht - nur der Aufsatz von Eileen Baldeschwiler: The Lyric Short Story. The Sketch of a History. In: Short Story Theories. Ed. by Charles May. Athens: Ohio University Press 1976, S. 202-213, genannt.

60

Vgl. besonders Stephan Fedler. Der Aphorismus. Begriffsspiel zwischen Philosophie und Poesie. Stuttgart: M \& P-Verlag für Wissenschaft und Forschung 1992 (= M \& P-Schriftenreihe für Wissenschaft und Forschung), Friedemann Spicker: Der Aphorismus. Begriff und Gattung von der Mitte des 18. Jahrhunderts bis 1912. Berlin/New York: de Gruyter 1997 (= Quellen und Forschungen zur Literatur- und Kulturgeschichte 11), F. S.: Studien zum deutschen Aphorismus im 20. Jahrhundert. Tübingen: Niemeyer 2000 (= Studien und Texte zur Sozialgeschichte der Literatur 79) und F. S.: Der deutsche Aphorismus im 20. Jahrhundert. Spiel, Bild, Erkenntnis. Tübingen: Niemeyer 2004.

61 Siehe hierzu die im Verzeichnis der Primärliteratur aufgelisteten Buchpublikationen. 
